\title{
SHARC II: a Caltech Submillimeter Observatory facility camera with 384 pixels
}

\author{
C. Darren Dowell ${ }^{a}$, Christine A. Allen ${ }^{b}$, Sachidananda Babu ${ }^{b}$, Minoru M. Freund ${ }^{b}$, \\ Matthew B. Gardner ${ }^{a}$, Jeffrey Groseth ${ }^{a}$, Murzy Jhabvala ${ }^{b}$, Attila Kovacs ${ }^{a}$, \\ Dariusz C. Lis ${ }^{a}$, S. Harvey Moseley, Jr. ${ }^{b}$, Thomas G. Phillips ${ }^{a}$, Robert Silverberg ${ }^{b}$, \\ George Voellmer ${ }^{b}$, and Hiroshige Yoshida ${ }^{c}$ \\ ${ }^{a}$ California Institute of Technology, Mail Code 320-47, 1200 E. California, Pasadena, CA 91125 \\ ${ }^{b}$ NASA-Goddard Space Flight Center, Greenbelt, MD 20771 \\ ${ }^{c}$ Caltech Submillimeter Observatory, 111 Nowelo St., Hilo, HI 96720
}

\begin{abstract}
SHARC II is a background-limited $350 \mu \mathrm{m}$ and $450 \mu \mathrm{m}$ facility camera for the Caltech Submillimeter Observatory undergoing commissioning in 2002. The key component of SHARC II is a $12 \times 32$ array of doped silicon 'pop-up' bolometers developed at NASA/Goddard. Each $1 \mathrm{~mm} \times 1 \mathrm{~mm}$ pixel is coated with a $400 \Omega$ /square bismuth film and located $\lambda / 4$ above a reflective backshort to achieve $>75 \%$ absorption efficiency. The pixels cover the focal plane with $>90 \%$ filling factor. At $350 \mu \mathrm{m}$, the SHARC II pixels are separated by $0.65 \lambda / \mathrm{D}$. In contrast to the silicon bolometers in the predecessor of SHARC II, each doped thermistor occupies nearly the full area of the pixel, which lowers the $1 / \mathrm{f}$ knee of the detector noise to $<0.03 \mathrm{~Hz}$, under load, at the bath temperature of $0.36 \mathrm{~K}$. The bolometers are AC-biased and read in 'total power' mode to take advantage of the improved stability. Each bolometer is biased through a custom $\sim 130 \mathrm{M} \Omega \mathrm{CrSi}$ load resistor at $7 \mathrm{~K}$ and read with a commercial JFET at $120 \mathrm{~K}$. The JFETs and load resistors are integrated with the detectors into a single assembly to minimize microphonic noise. Electrical connection across the $0.36 \mathrm{~K}$ to $4 \mathrm{~K}$ and $4 \mathrm{~K}$ to $120 \mathrm{~K}$ temperature interfaces is accomplished with lithographed metal wires on dielectric substrates. In the best $25 \%$ of winter nights on Mauna Kea, SHARC II is expected to have an NEFD at $350 \mu \mathrm{m}$ of $1 \mathrm{Jy} \mathrm{Hz}^{-1 / 2}$ or better. The new camera should be at least 4 times faster at detecting known point sources and 30 times faster at mapping large areas compared to the prior instrument.
\end{abstract}

Keywords: submillimeter, instrumentation, bolometers

\section{INTRODUCTION}

Within the past decade, submillimeter and millimeter continuum studies have made an increasingly significant contribution to astronomy as new, more complex instruments with more and more detectors have raised the standard of mapping speed (e.g., SHARC, ${ }^{1}$ SCUBA, ${ }^{2}$ MAMBO,,${ }^{3,4}$ BOOMERANG, ${ }^{5}$ MAXIMA ${ }^{6}$ ). Most (sub)millimeter detectors use conical feedhorns or Winston concentrators to funnel light from relatively large entrance apertures onto bolometers with relatively small extent. In this paper, we describe a contrasting approach which led to the development, at NASA/Goddard Space Flight Center, of a 'bare', filled two-dimensional bolometer array for a ground-based $350 \mu \mathrm{m}$ and $450 \mu \mathrm{m}$ camera (SHARC II) and soon will lead to one for a SOFIA camera $\left(\mathrm{HAWC}^{7}\right)$. SHARC II commissioning began in 2002, and early results are described here.

Filled focal plane arrays with instantaneous Nyquist sampling offer optimal mapping speed when the desired field of view can be completely covered with detectors. ${ }^{8}$ Not having multiplexed readouts, the filled arrays for SHARC II and HAWC do not cover all of the available telescope field of view, suggesting that they work best for mapping compact intensity distributions. However, the relatively large number of pixels which has been achieved $(>300)$ also makes SHARC II competitive for mapping large areas compared to other instruments with fewer pixels (e.g., 91 for SCUBA at $450 \mu \mathrm{m}$ ) but larger coverage per pixel.

Further author information:

C.D.D.: E-mail: cdd@submm.caltech.edu, Telephone: 16263956675 


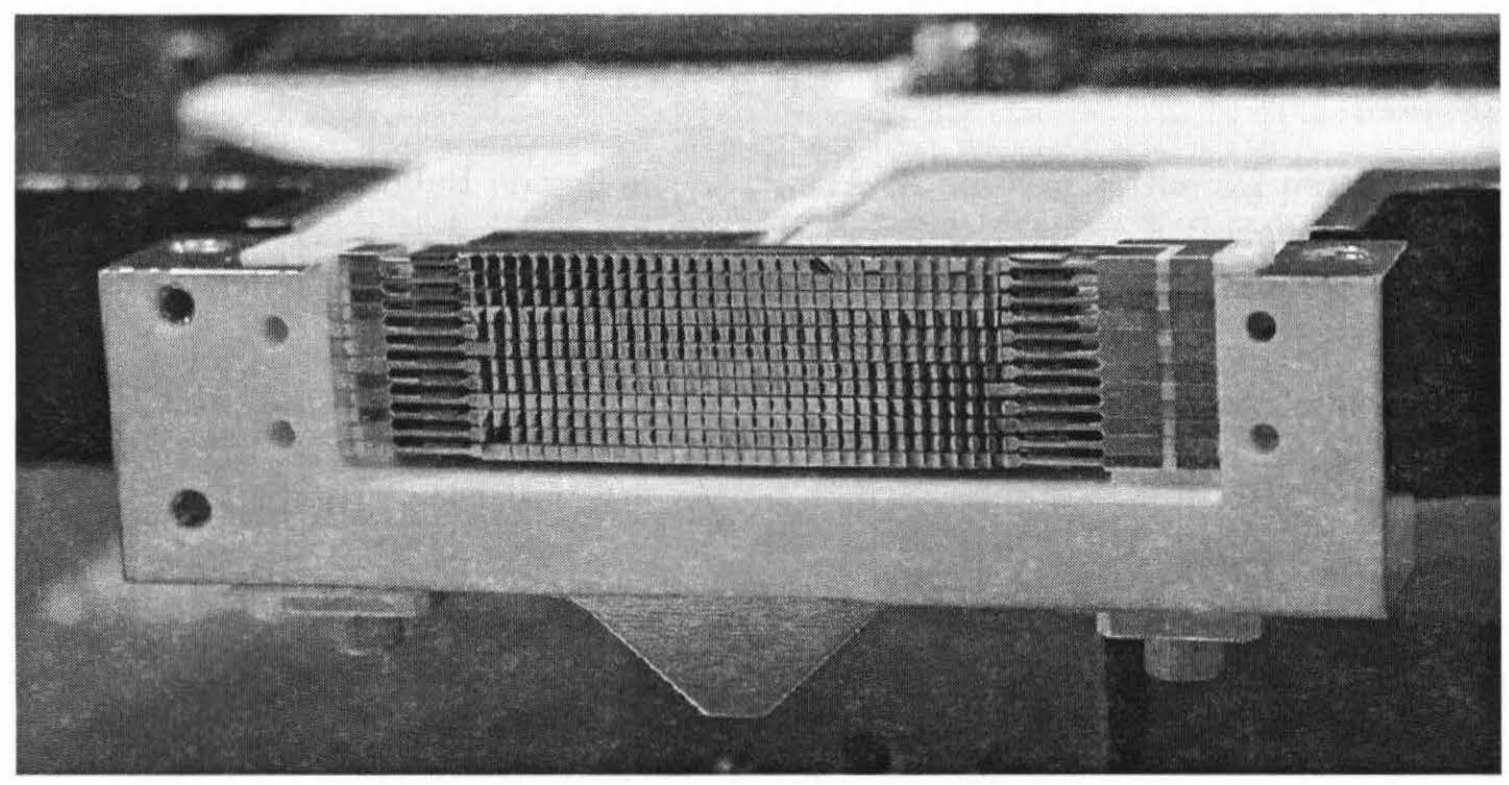

Figure 1. The SHARC II focal plane near the end of the assembly process. The bolometers cover an area of $32 \mathrm{~mm} \times$ $12 \mathrm{~mm}$.

Compared to arrays with feedhorns or concentrators, bare focal plane arrays are more vulnerable to loading by radiation from the cryostat itself. Each $1 \mathrm{~mm}^{2}$ SHARC II bolometer has approximately a $2 \pi$ steradian view of cryostat components at $4 \mathrm{~K}$ and a $2 \pi$ steradian view of components at $0.3 \mathrm{~K}$. Taking a worst case that it is perfectly absorptive at all wavelengths, it would experience a load of $15 \mathrm{pW}$ from the cryostat, resulting in an NEP* of $8 \times 10^{-17} \mathrm{~W} \mathrm{~Hz}^{-1 / 2}$. (In fact, half of this load is observed; see §7.1.) In operation at $350 \mu \mathrm{m}, \mathrm{a}$ SHARC II bolometer sees a background of $120-200 \mathrm{pW}$ and $6-10 \times 10^{-16} \mathrm{~W} \mathrm{~Hz}^{-1 / 2}$ from the sky, rendering the load from the cryostat negligible. In principle, the cryostat load can be reduced to $\leq 0.3 \mathrm{pW}$ and $7 \times 10^{-18} \mathrm{~W} \mathrm{~Hz}^{-1 / 2}$ by pumping the liquid helium down to $1.5 \mathrm{~K}$.

Of greater concern for bare arrays is stray radiation from warmer objects (e.g., JFETs, outer layers of the cryostat, poorly heatsunk shafts and cables) that is not adequately intercepted. In SHARC II, we have observed a small leak of JFET radiation which has no significant effect on instrument performance (§7.1) and find no evidence of additional light leaks.

\section{PROTOTYPE $12 \times 32$ POP-UP BOLOMETER ARRAY}

The mechanical configuration of the SHARC II bolometer array is described by Voellmer et al. in these proceedings. ${ }^{9}$ Briefly, the $12 \times 32$ bolometer array (Figure 1), load resistors, and thermally-isolated JFETs are housed in a structure approximately $18 \mathrm{~cm} \times 17 \mathrm{~cm} \times 18 \mathrm{~cm}$ high, having a total mass of $5 \mathrm{~kg}$, and heat sunk to $4 \mathrm{~K}$. A copper cable (terminated with a copper block to be heat sunk to $0.3 \mathrm{~K}$ ) and four terminals (to dump the JFET power to $4 \mathrm{~K}$ ) protrude from the housing. The outputs of the JFETs terminate in 6 customized connectors with 152 pins each (Packard-Hughes/Delphi Connection Systems HDPC-P152 series). Additional thermometry terminates in two 25-pin micro-D connectors.

\subsection{Basic mechanical, electrical, and thermal properties of the bolometers}

Each bolometer is fabricated on a $1 \mu \mathrm{m}$ silicon membrane and has a collecting area of $1 \mathrm{~mm} \times 1 \mathrm{~mm}$ (Figure 2). The full area is ion implanted with phosphorus and boron, to a depth of $\sim 0.4 \mu \mathrm{m}$, to form a thermistor; maximizing the thermistor area minimizes the bolometer $1 / \mathrm{f} \mathrm{noise}^{10}$ Electrical contact between the thermistor

\footnotetext{
*In this paper, NEP refers to the electrical NEP - the noise detected in the bolometer.
} 


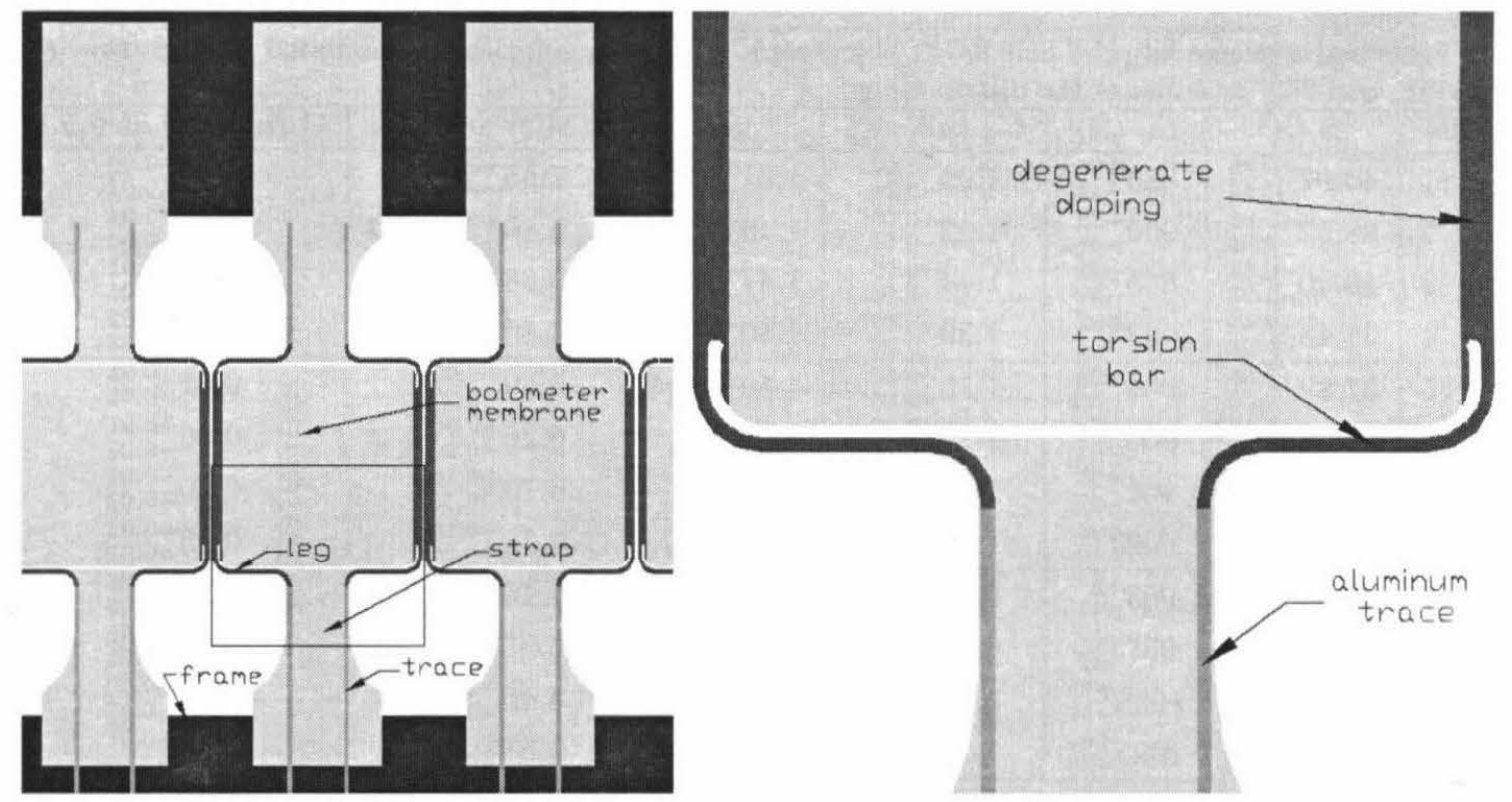

Figure 2. Schematic diagram of unfolded SHARC II bolometers. The bolometer ( $1 \mathrm{~mm}$ square), legs, and strap are patterned from $1 \mu \mathrm{m}$ silicon membrane (left view). The bolometers are anchored to the thick silicon frame at the ends of the straps. Most of the top surface of the bolometer is doped to create a thermistor, and most of the back surface is coated with an absorbing bismuth film. Two edges of each bolometer and the legs are degenerately doped to make electrical contact with the thermistor (right view). Aluminum traces overlap the degenerate traces on the strap and proceed to wire bond pads at the edge of the silicon frame.

and aluminum traces on the silicon frame is accomplished with degenerately-doped leads on the edge of the thermistor and running down the bolometer legs. Each of the four thermally-isolating legs is $16 \mu \mathrm{m}$ wide and $420 \mu \mathrm{m}$ long.

Each row of 32 bolometers is a continuous silicon structure, and each $10 \mathrm{~cm}$ silicon wafer contains 16 rows. Twelve wafers with three target implant doses (net doping density $\approx 2 \times 10^{18} \mathrm{~cm}^{-3}$ ) were processed to produce the SHARC II array. Six of the wafers had components within the desired resistance range. ${ }^{11} 18$ rows from three wafers advanced to the folding stage ${ }^{9}$ resulting in an inventory of 16 succesfully-folded rows. Twelve rows were selected to build SHARC II following their measurement at $0.3 \mathrm{~K}{ }^{12}$

Prior to folding, each bolometer is coated with an absorbing $\sim 200 \AA$ bismuth film and a protective $\sim 160 \AA$ $\mathrm{SiO}$ film. Witness samples coated in the same evaporations were measured at $4 \mathrm{~K}$ to verify that the target resistance of $400 \Omega$ /square was produced; an accuracy of $\pm 40 \Omega$ /square was achieved. The $400 \Omega$ /square absorber in concert with the $100 \mu \mathrm{m}$ spacing between the bolometer and reflective busbar edge ${ }^{9}$ theoretically produces $100 \%$ absorption at $\lambda=400 \mu \mathrm{m}$ and $>98 \%$ absorption at $\lambda=350 \mu \mathrm{m}$ and $450 \mu \mathrm{m}$.

Dark IV curves were measured in the Caltech lab for all bolometers in the assembled array at base temperatures of $0.30 \mathrm{~K}, 0.50 \mathrm{~K}$, and $0.88 \mathrm{~K}^{\dagger}$. The IV curves for each bolometer were fit with a standard model for the resistance $R$ and thermal conductance $G=d P / d T$ as a function of temperature $T$ :

$$
\mathrm{R}=\mathrm{R}_{0} \exp \sqrt{\Delta / \mathrm{T}}, \mathrm{G}=\mathrm{G}_{0}(\mathrm{~T} / 1 \mathrm{~K})^{\beta} .
$$

Fits to the data are summarized in Table 1. Since the characteristics of the rows were known prior to stacking them, it was possible to give priority toward the center of the array to rows with higher and more uniform resistance, higher yield, and a more uniform separation of bolometer and backshort.

\footnotetext{
${ }^{\dagger}$ To reduce internal radiation loads, the IV curves were obtained with the liquid helium pumped to $1.5 \mathrm{~K}$ and with only one JFET drawer active with a lowered operating temperature of $\sim 75 \mathrm{~K}$. See $\S 7.1$.
} 
Table 1. Measured characteristics of SHARC II bolometers. Median quantities are tabulated for each row, and 'error bars' give $75 \%$ and $25 \%$ quartiles of the distributions.

\begin{tabular}{|r|c|c|c|c|c|c|}
\hline Row & $\Delta(\mathrm{K})$ & $\mathrm{R}_{0}(\Omega)$ & $\mathrm{G}_{0}(\mathrm{nW} / \mathrm{K})$ & $\beta$ & $\mathrm{R}(\mathrm{M} \Omega)$ at $0.5 \mathrm{~K}$ & $\mathrm{G}(\mathrm{nW} / \mathrm{K})$ at $0.5 \mathrm{~K}$ \\
\hline 1 & $40.97_{-1.40}^{+0.85}$ & $883_{-45}^{+28}$ & $1.09_{-0.04}^{+0.04}$ & $1.61_{-0.01}^{+0.03}$ & $7.55_{-0.83}^{+0.34}$ & $0.36_{-0.01}^{+0.00}$ \\
2 & $38.52_{-2.86}^{+0.86}$ & $930_{-41}^{+38}$ & $1.22_{-0.08}^{+0.05}$ & $1.46_{-0.01}^{+0.01}$ & $5.86_{-1.33}^{+0.51}$ & $0.45_{-0.04}^{+0.01}$ \\
3 & $40.50_{-1.02}^{+0.36}$ & $865_{-20}^{+21}$ & $1.44_{-0.04}^{+0.05}$ & $1.47_{-0.02}^{+0.01}$ & $6.88_{-0.51}^{+0.43}$ & $0.52_{-0.01}^{+0.01}$ \\
4 & $35.48_{-0.28}^{+2.45}$ & $993_{-54}^{+82}$ & $1.39_{-0.07}^{+0.07}$ & $1.50_{-0.03}^{+0.02}$ & $4.80_{-0.27}^{+0.94}$ & $0.50_{-0.03}^{+0.02}$ \\
\hline 5 & $37.89_{-0.50}^{+0.36}$ & $955_{-52}^{+29}$ & $1.15_{-0.05}^{+0.05}$ & $1.50_{-0.03}^{+0.01}$ & $5.69_{-0.22}^{+0.07}$ & $0.41_{-0.02}^{+0.01}$ \\
6 & $39.13_{-0.52}^{+0.15}$ & $900_{-8}^{+14}$ & $1.29_{-0.06}^{+0.04}$ & $1.49_{-0.01}^{+0.01}$ & $6.26_{-0.26}^{+0.05}$ & $0.46_{-0.02}^{+0.01}$ \\
7 & $39.46_{-0.90}^{+0.64}$ & $902_{-22}^{+26}$ & $1.17_{-0.08}^{+0.05}$ & $1.54_{-0.01}^{+0.03}$ & $6.70_{-0.78}^{+0.16}$ & $0.40_{-0.03}^{+0.02}$ \\
8 & $39.72_{-0.55}^{+0.21}$ & $909_{-10}^{+23}$ & $1.22_{-0.11}^{+0.05}$ & $1.51_{-0.02}^{+0.02}$ & $6.78_{-0.26}^{+0.11}$ & $0.43_{-0.03}^{+0.02}$ \\
\hline 9 & $40.84_{-0.43}^{+0.44}$ & $903_{-11}^{+8}$ & $0.93_{-0.01}^{+0.02}$ & $1.53_{-0.02}^{+0.03}$ & $7.46_{-0.27}^{+0.55}$ & $0.32_{-0.00}^{+0.01}$ \\
10 & $39.30_{-0.48}^{+0.32}$ & $927_{-17}^{+8}$ & $0.95_{-0.05}^{+0.07}$ & $1.46_{-0.01}^{+0.02}$ & $6.53_{-0.28}^{+0.25}$ & $0.35_{-0.03}^{+0.02}$ \\
11 & $32.50_{-0.37}^{+3.23}$ & $1068_{-87}^{+25}$ & $0.86_{-0.03}^{+0.02}$ & $1.45_{-0.05}^{+0.05}$ & $3.46_{-0.16}^{+1.16}$ & $0.32_{-0.02}^{+0.00}$ \\
12 & $30.99_{-1.46}^{+2.85}$ & $1095_{-85}^{+44}$ & $1.21_{-0.11}^{+0.02}$ & $1.42_{-0.02}^{+0.03}$ & $2.87_{-0.37}^{+0.90}$ & $0.45_{-0.03}^{+0.01}$ \\
\hline
\end{tabular}

The typical electrical time constant and heat capacity measured in the dark for a subset of the bolometers are approximately $6 \mathrm{msec}$ and $4 \mathrm{pJ} / \mathrm{K}$ at $0.5 \mathrm{~K} .{ }^{13}$ The heat capacity is not well understood and is a factor of two higher than expected. Assuming the heat capacity is proportional to $\mathrm{T}^{1}$, the expected time constant under the operating $350 \mu \mathrm{m}$ load ( $\S 1$ and $\S 7.2)$ is also $6 \mathrm{msec}$, although this has not yet been confirmed experimentally.

\subsection{Responsivity and sensitivity}

At a base temperature of $0.36 \mathrm{~K}$ and in the dark, the bolometers have a peak responsivity of approximately $4 \times 10^{8} \mathrm{~V} / \mathrm{W}$ and a minimum NEP of approximately $6 \times 10^{-17} \mathrm{~W} \mathrm{~Hz}^{-1 / 2}$ at $10 \mathrm{~Hz}$. Phonon noise is the dominant contributor, followed by bolometer Johnson noise. An NEP of $5 \times 10^{-17} \mathrm{~W} \mathrm{~Hz}^{-1 / 2}$ has been measured and reported previously for SHARC II bolometers operated at $0.30 \mathrm{~K} .^{13}$

When the lab $350 \mu \mathrm{m}$ radiation load of $230 \mathrm{pW}$ is applied $(\S 7.2)$, the bolometers warm up to $\sim 0.79 \mathrm{~K}$ without bias power and $\sim 0.85 \mathrm{~K}$ with bias. The peak responsivity drops to $2 \times 10^{7} \mathrm{~V} / \mathrm{W}$, and the detector NEP rises to $3 \times 10^{-16} \mathrm{~W} \mathrm{~Hz}^{-1 / 2}$. Smaller than the photon noise from the radiation load, the bolometer Johnson noise and phonon noise are about equal to amplifier noise since the bolometer resistance is small $(\sim 0.8 \mathrm{M} \Omega)$.

\section{COLD READOUT COMPONENTS}

\subsection{Load resistors}

The bolometers are operated with an approximate current bias using a large load resistor. Space at $0.3 \mathrm{~K}$ is limited, so the load resistors are located in the readout drawers which are heat sunk to the liquid helium bath and rise to $7 \mathrm{~K}$ when the JFETs are heated. ${ }^{9}$ The intrinsic Johnson noise $\sqrt{4 \mathrm{kTR}_{\mathrm{L}}}$ of the load resistors is high, but the voltage noise at the common terminal with the bolometer is reduced by the factor $\mathrm{Z} /\left(\mathrm{Z}+\mathrm{R}_{\mathrm{L}}\right)$, where $\mathrm{Z}(\omega)$ is the bolometer dynamic impedance. For SHARC II, Z $(0) \approx 1 \mathrm{M} \Omega$ and $\mathrm{R}_{\mathrm{L}} \approx 130 \mathrm{M} \Omega$.

The large value resistors are made custom for SHARC II and HAWC at Goddard. The resistive element is a chromium silicide film with a sheet resistance $\sim 1100 \Omega$ /square sputtered on a fused quartz substrate. ${ }^{14}$ Given the details of the lithographed serpentine patterns and bond pads, a packaging density of $\sim 20 \mathrm{M} \Omega / \mathrm{mm}^{2}$ was achieved. The full value resistances were targeted for HAWC, which has a larger resistance requirement; for SHARC II, a lithographed tap at $1 / 3$ of the full length and resistance is used. 


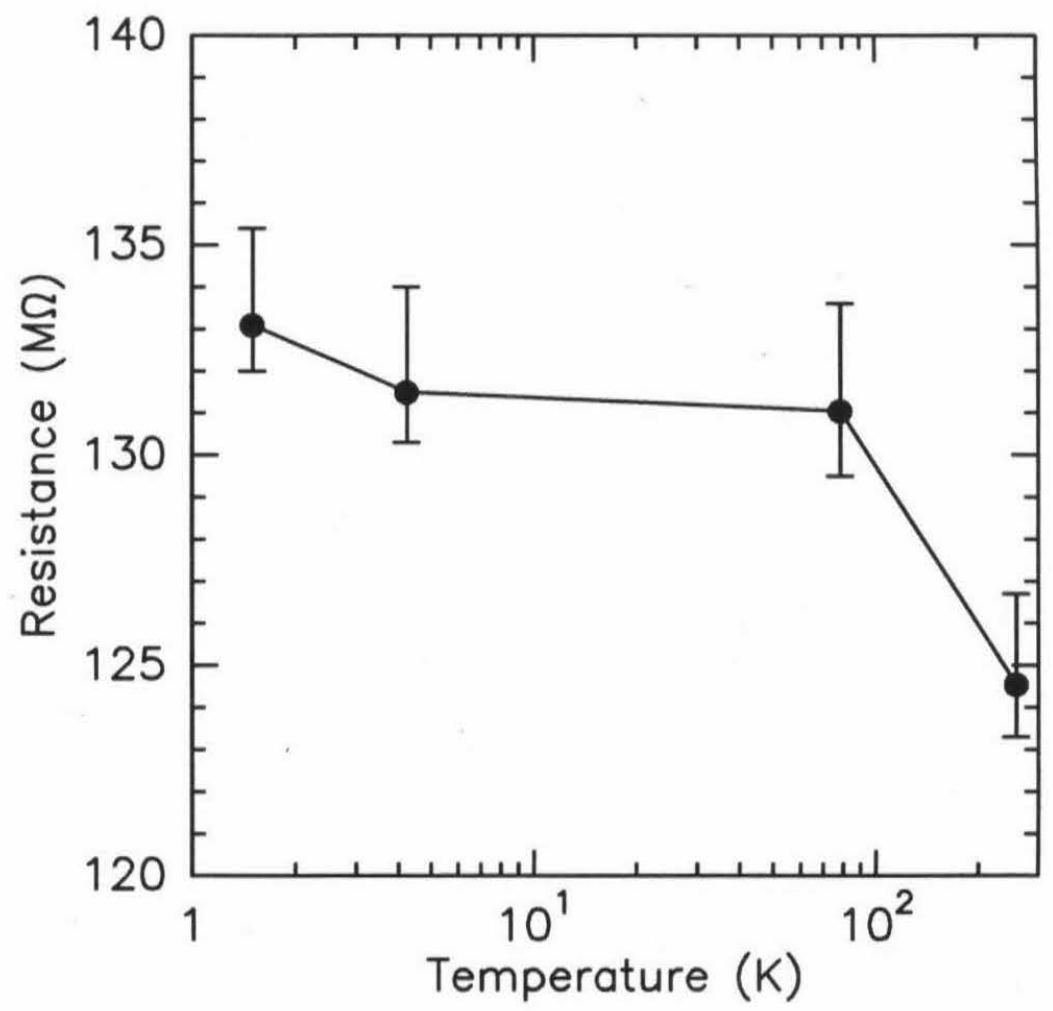

Figure 3. Resistance vs. temperature for CrSi load resistors in SHARC II. The resistances of a sample of 22 load resistors servicing row 6 are shown. The points show the medians, and the error bars show the 25 and 75 percentiles. The actual operating temperature is $7 \mathrm{~K}$.

The resistors are fabricated in rows of many long, (mechanically) parallel resistors. The rows are probed and diced to produce blocks of 1 to 32 functional resistors, which are then sorted to produce groups of 32 matched to $\sim 10 \%$ in resistance.

The resistance of the $\mathrm{CrSi}$ film is only weakly dependent on temperature (Figure 3). At operating temperature, the median value of all of the load resistors in SHARC II is $132 \mathrm{M} \Omega$. As demonstrated by the complete system stability (Figure 7), the CrSi load resistors have sufficient resistance stability for this application.

\subsection{JFETs}

The JFETs in SHARC II and HAWC are commercially available die (InterFET NJ132L) operated in a commondrain voltage follower configuration. At $120 \mathrm{~K}$ and operated with low power $(50 \mu \mathrm{A}$ drain current, $2 \mathrm{~V}$ drain voltage), they have a typical noise of $5 \mathrm{nV} \mathrm{Hz}-1 / 2$ at $3 \mathrm{~Hz}$ and $\lesssim 3 \mathrm{nV} \mathrm{Hz}^{-1 / 2}$ at $100 \mathrm{~Hz}$. At this same power, the JFETs have an output impedance $\mathrm{dV} / \mathrm{dI}$ of $\sim 1 \mathrm{k} \Omega$. The JFETs are packaged on ceramic substrates inside a readout drawer with internal thermal isolation. ${ }^{9}$

\subsection{Lithographed wire bridges}

To manage the $\sim 1000$ wires from $0.3 \mathrm{~K}$ to $4 \mathrm{~K}$ and from $4 \mathrm{~K}$ to $120 \mathrm{~K}$, the SHARC II array uses the Goddard microbridge (or 'bridge chip') technology. ${ }^{9}$ Each microbridge wire consists of a metal trace evaporated on a freestanding, serpentine polyimide filament with width $20 \mu \mathrm{m}$, thickness $2 \mu \mathrm{m}$, and total length $3 \mathrm{~mm}$. Each of the 1176 microbridge wires inside the JFET drawers has resistances of $\sim 57 \Omega, \sim 18 \Omega$, and $\sim 10 \Omega$ at room temperature, $120 \mathrm{~K}$, and $4 \mathrm{~K}$, respectively; the metal layer is aluminum. Each of the 840 microbridge wires from the detector at $0.3 \mathrm{~K}$ to the fanout boards at $4 \mathrm{~K}$ has a room temperature resistance of $\sim 3300 \Omega$; the metal is titanium. 


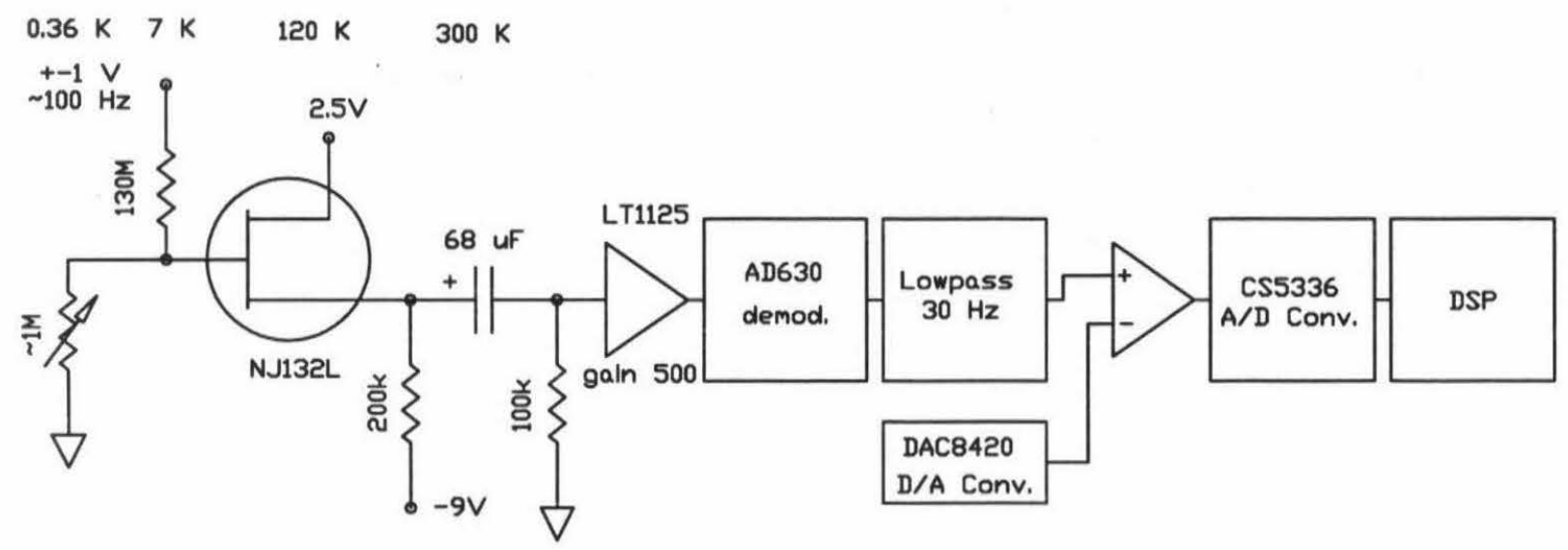

Figure 4. SHARC II readout electronics. An approximately square wave bias produces an approximately square wave signal at the JFET and output of the first operational amplifier (op amp). The AD 630 demodulator rectifies the signal, producing a large DC component with glitches at the transitions of the bias and demodulation references. The glitches are heavily filtered prior to the last op amp stage, which has selectable gains of 2.8 and 21 . At the same stage, a digital-to-analog converter (DAC) subtracts away most of the DC signal. The DAC is held fixed over an integration or sequence of integrations.

\section{CABLING AND WARM READOUT COMPONENTS}

\subsection{Electronics overview}

Flat, flexible cables lithographed on Kapton film connect the Packard-Hughes connectors inside the detector housing to 100-pin micro-D connectors on a cryostat bulkhead, all of which are at the same temperature of 4 $\mathrm{K}$. Starting at the $4 \mathrm{~K}$ bulkhead, $0.1 \mathrm{~mm}$ manganin wires woven with Nomex into a ribbon cable assembly 1.4 $\mathrm{m}$ in length (Tekdata, Ltd.) carry the signals to a hermetic 79-pin connector at the cryostat wall. The cables are heat sunk midway to the He-vapor-cooled station at $\sim 20 \mathrm{~K}(\S 5)$. Following the hermetic connector, each signal proceeds to an amplifier circuit board within an aluminum enclosure with an intermediate RFI-filtered connector (Spectrum Control SCI-56-641-019, $4 \mathrm{nF}$ ) on an internal bulkhead. The aluminum enclosures are attached rigidly to the cryostat. Following amplification and additional analog processing, each signal passes through another RFI-filtered connector (Spectrum Control SCI-56-732-005, $4 \mathrm{nF}$ pi) on the wall of the amplifier enclosure and via a cable to a custom A/D system based on the Crystal Semiconductor/Cirrus Logic CS5336 chip. Digitized signals are passed through fiber optic cables to a commercial DSP board (Pentek 4290) in a VME-format computer with a SPARC CPU (Themis Computer).

\subsection{AC-biased total-power amplifiers}

The SHARC II amplifiers are an implementation of an AC-biased 'total-power' readout, ${ }^{5}$ in this case having unbalanced, single-ended signals (one JFET and load resistor per bolometer); square-wave modulation and demodulation; and no intrinsic high-pass filtering (Figure 4). The demodulation reference signal is delayed digitally from the bias reference by an amount programmable for each row independently. The DC voltage of the bolometer is zeroed in hardware by a digital-to-analog converter. The Analog Devices DAC8420 had the lowest noise of several DACs that we tested; however, it is still advantageous to have as much gain in the first-stage op amp as possible to minimize the effect of the DAC $1 / \mathrm{f}$ noise. For most observing sequences, which are differential in nature, the value of the DAC does not enter the analysis. However, the programmed value of the DAC can be used for radiometric measurements (§8.3). To date, the standard bias frequency is $125 \mathrm{~Hz}$.

The output signal for a bolometer read in total-power mode responds with full gain to radiation modulated at frequencies from the low-pass cutoff $(\sim 30 \mathrm{~Hz})$ down to DC with (in principle) low 1/f noise. 


\section{CRYOSTAT}

The SHARC II cryostat (custom built by Precision Cryogenic Systems, Inc.) is $47 \mathrm{~cm}$ in diameter, $104 \mathrm{~cm}$ in length, and provides an enclosed $4 \mathrm{~K}$ work volume $35 \mathrm{~cm}$ in diameter and $51 \mathrm{~cm}$ in length. A liquid helium reservoir with an estimated volume of 10.5 liters cools the $4 \mathrm{~K}$ shield and accepts the load from the JFETs. A 7.5 liter liquid nitrogen reservoir cools an $\sim 80 \mathrm{~K}$ radiation shield. Mechanical support of the reservoirs and shịelds is provided by large G10 fiberglass cylinders and Kevlar cords. Cooling power of the helium vapor is utilized, via a small heat exchanger part way up the single helium fill/vent tube, to cool electronic cables and the support cylinder for the helium reservoir. A compact, closed-cycle ${ }^{4} \mathrm{He} /{ }^{3} \mathrm{He}$ refrigerator ${ }^{15}$ (Dr. S. T. Chase, Chase Research) provides $2 \mathrm{~J}$ of cooling capacity at $0.35 \mathrm{~K}$ for the bolometer array and is recycled under computer control without pumping on the cryostat. Cycles last $20+$ hours.

With the JFET electronics off, the parasitic load on the liquid helium is $78 \mathrm{~mW}$, giving a hold time of 100 hours. A power of $83 \mathrm{~mW}$ is required to bring all 384 JFETs to their operating temperature of $120 \mathrm{~K}$, which doubles the liquid helium consumption. Following a $12+$ hour liquid nitrogen precool, approximately 35 liters of liquid helium are required to cool down the $4 \mathrm{~K}$ hardware. The suspended portion of the detector, ${ }^{4} \mathrm{He} /{ }^{3} \mathrm{He}$ refrigerator coldhead, and interfacing cold bar cool passively and effectively during the liquid nitrogen precool, but the ${ }^{3} \mathrm{He}$ charcoal pump must be heated to achieve cooling to $4 \mathrm{~K}$. The liquid nitrogen hold time is approximately 45 hours.

The helium vapor heat exchanger is a $2.5 \mathrm{~cm}$ long copper block in series with the stainless steel tube to the reservoir and the one to the vacuum wall, all with inner diameter $1.3 \mathrm{~cm}$. The copper block is attached with copper strips to an aluminum plate which joins the fiberglass cylinder from the liquid nitrogen reservoir with the one supporting the liquid helium reservoir. The electronic cables are epoxied to copper tabs and also attached to the vapor-cooled plate. The plate reaches an equilibrium temperature of $20 \mathrm{~K}$ with the detector electronics off and $13 \mathrm{~K}$ with the JFETs at $120 \mathrm{~K}$.

\section{OPTICS}

The SHARC II bolometer array is located directly at the $\mathrm{f} / 4.48$ focus of the CSO Cassegrain relay optics. ${ }^{16,17}$ The pixel separation of $1 \mathrm{~mm}$ corresponds to $4.6^{\prime \prime}$ on the sky, or $0.65 \lambda / \mathrm{D}$ at $350 \mu \mathrm{m}$ (nearly Nyquist sampled). The imaging in the geometrical limit for the secondary mirror vertex on axis was analyzed with Code V (Optical Research Associates). For the detector array oriented in azimuth, the mean Strehl ratio over the array is 0.97 with a minimum of 0.94 . The imaging is somewhat worse with the array in elevation; the mean Strehl ratio is 0.93 with a minimum of 0.88 . The distortion (maximum deviation from a regular grid) is $<0.3 \mathrm{~mm}$.

Rejection of radiation outside the envelope of geometrical rays is accomplished with a series of baffles with circular or rectangular apertures (Figure 5). Filtering is performed with a combination of interference filters, crystals, and plastics (Table 2). A filter wheel selects among $350 \mu \mathrm{m}, 450 \mu \mathrm{m}$, and $850 \mu \mathrm{m}$ bandpass filters and a blank (dark) position. The $350 \mu \mathrm{m}$ filter is encoded with a stationary magnetoresistor sensor (Siemens/Infineon FP210L100-22) actuated by an iron finger on the filter wheel. The bolometers should have background-limited performance at $350 \mu \mathrm{m}$ and $450 \mu \mathrm{m}$, but should be limited by detector noise at $850 \mu \mathrm{m}$. Most testing has employed the $350 \mu \mathrm{m}$ bandpass (Figure 6), which is the optimization wavelength of the instrument.

\section{SHARC II ARRAY PERFORMANCE}

The thermal performance of the SHARC II array meets all requirements for operation at the CSO. ${ }^{9}$ The impact of the detector on the ${ }^{3} \mathrm{He}$ lifetime is negligible, the $83 \mathrm{~mW}$ dissipation of the JFETs into the liquid helium can be managed, and the load resistors (at $7 \mathrm{~K}$ when the JFETs are on) contribute $\leq 3 \%$ of the total detector readout noise even in the dark.

The combined yield of the bolometers, load resistors, and JFETs is $\sim 85 \%$, with the greatest losses likely occuring on the fanout boards between the bolometers and readout drawers. ${ }^{9}$ Most of the problems are difficult or impossible to repair. 

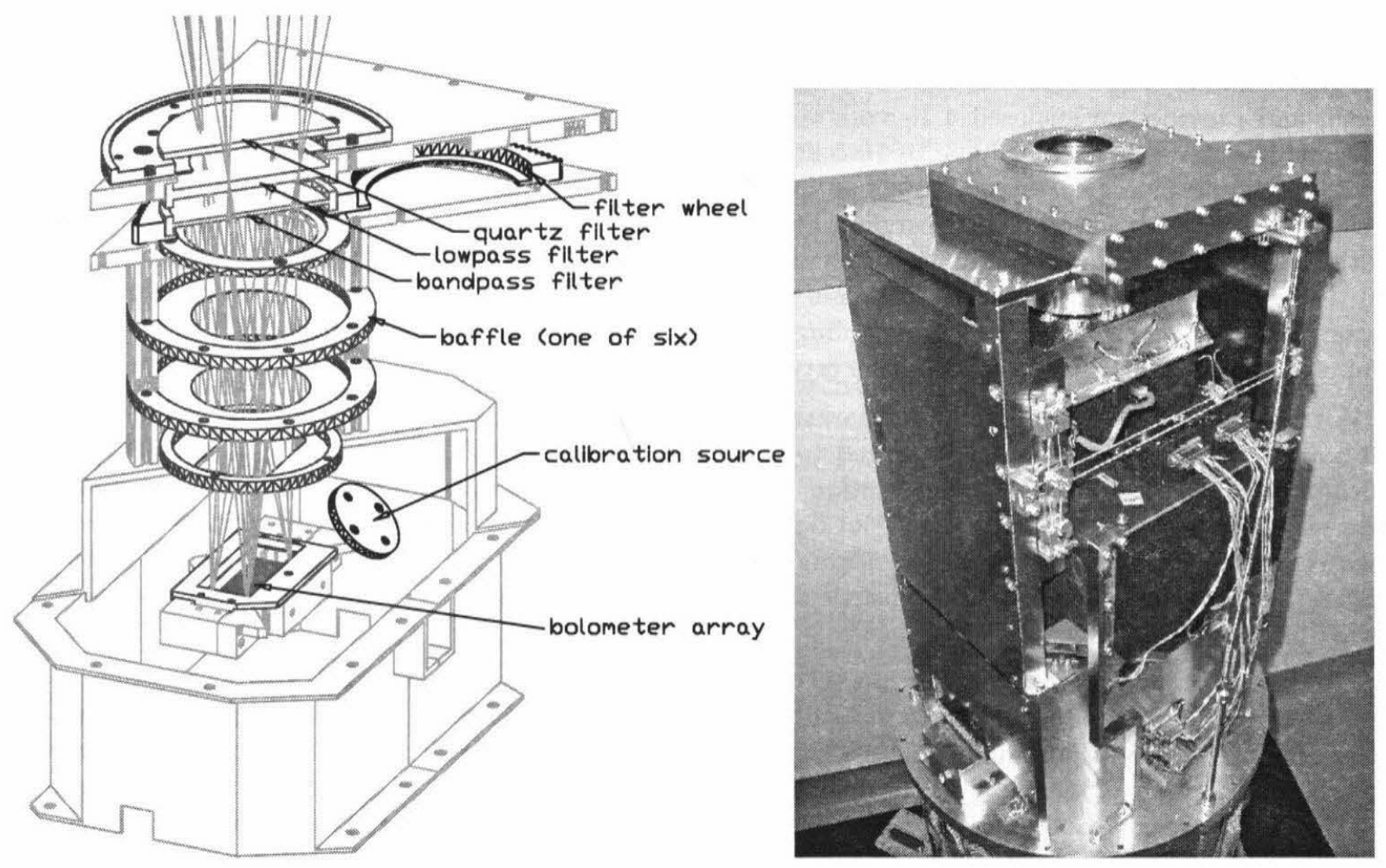

Figure 5. Filtering and field-of-view restricting optics in SHARC II (left view). Many elements are displayed in crosssection. Photograph of SHARC II $4 \mathrm{~K}$ apparatus (right view).

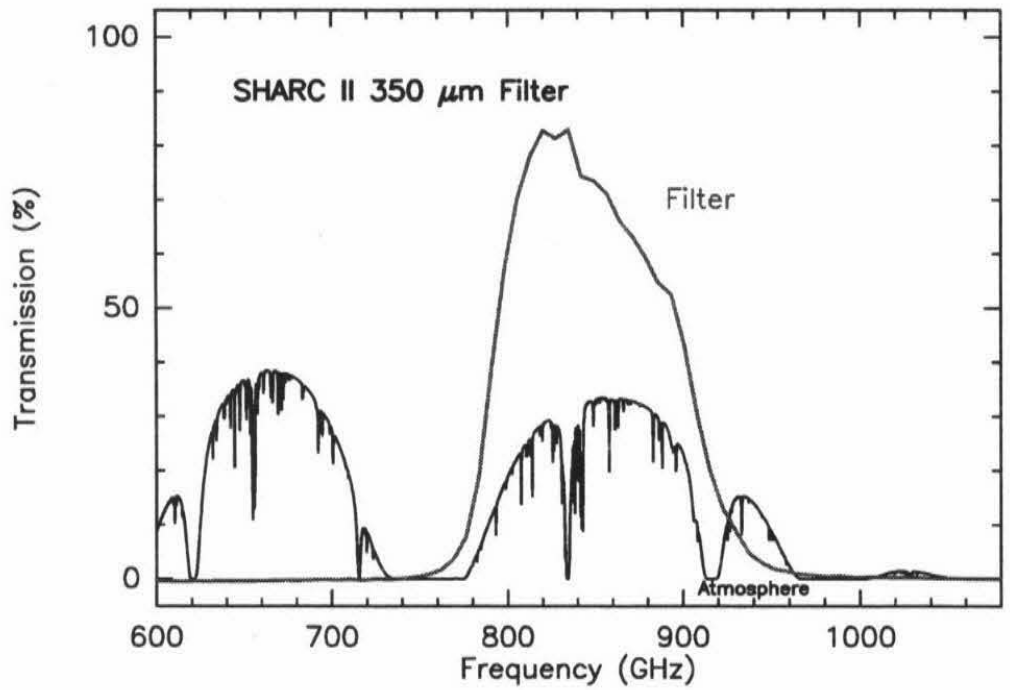

Figure 6. Transmission spectrum of $350 \mu \mathrm{m}$ filter system compared to the atmospheric transmission. The filter spectrum was derived by measuring each of the filter components (Table 2) individually at operating temperature with a Fourier transform spectrometer. The atmospheric spectrum is a model ${ }^{18}$ for $\operatorname{CSO} \tau_{225 \mathrm{GHz}}=0.049$, the first quartile of the opacity distribution for winter nights. Assuming the detector is nearly perfectly absorptive $(\S 7.2)$, the system quantum efficiency is essentially the same as the filter curve. 
Table 2. Summary of SHARC II Optics.

\begin{tabular}{|c|c|}
\hline Parameter/Item & Value/Composition \\
\hline f/ ratio & $\mathrm{f} / 4.5$ \\
\hline pixel size & $4.6^{\prime \prime} \times 4.6^{\prime \prime}$ \\
\hline field of view & 2.3 square arcminutes \\
\hline angular resolution, $350 \mu \mathrm{m}$ & $9^{\prime \prime}$ FWHM \\
\hline mean Strehl ratio, $350 \mu \mathrm{m}$ & 0.95 \\
\hline window & $1 \mathrm{~mm}$ high-density polyethylene \\
\hline $77 \mathrm{~K}$ filter & $\begin{array}{c}\text { z-cut crystal quartz, } 2 \mathrm{~mm} \\
\text { front surface: } 58 \mu \mathrm{m} \text { clear polyethylene } \\
\text { back surface: } 50 \mu \mathrm{m} \text { black poly, } 8 \mu \mathrm{m} \text { clear poly }\end{array}$ \\
\hline $\begin{array}{c}4 \mathrm{~K} \\
\text { filters }\end{array}$ & $\begin{array}{c}\text { quartz (same specifications) } \\
33 \mathrm{~cm}^{-1} \text { lowpass (Cochise Instruments/P. Ade) }\end{array}$ \\
\hline $\begin{array}{c}4 \mathrm{~K} \\
\text { bandpass filters } \\
\text { (Cochise Instruments/P. Ade) }\end{array}$ & $\begin{array}{l}350 \mu \mathrm{m}, \Delta \lambda(\text { FWHM }) / \lambda=0.13 \\
450 \mu \mathrm{m}, \Delta \lambda(\text { FWHM }) / \lambda=0.10 \\
850 \mu \mathrm{m}, \Delta \lambda(\text { FWHM }) / \lambda=0.08\end{array}$ \\
\hline
\end{tabular}

\subsection{Limits to sensitivity}

In recent months of testing, we have observed some limits to the sensitivity of the SHARC II array as built and as employed. By choosing to have a $4 \mathrm{~K}$ filter wheel as opposed to a single $0.36 \mathrm{~K}$ bandpass filter capping the bolometer array, and by choosing to operate the cryostat without pumping on the liquid helium to simplify operation at the observatory, we expose the bolometers to $4 \mathrm{~K}$ radiation. By comparing bolometer IV curves with the cryostat at $4 \mathrm{~K}$ and with the cryostat at $1.5 \mathrm{~K}$ (for which the radiation is significantly reduced), we find a difference in power of $8 \mathrm{pW}$. This load could potentially be reduced by constructing a $0.3 \mathrm{~K}$ baffle to limit the field of view of the detectors to the incoming beam or by adding a $0.3 \mathrm{~K}$ high-pass filter to reject cryostat radiation at $\lambda>500-1000 \mu \mathrm{m}$.

We also detect a small radiation leak from the JFETs. When all three JFET substrates are at operating temperatures of $120 \mathrm{~K}$, an excess power of $4 \mathrm{pW}$ is observed in IV curves. Not all surfaces near the detector and JFETs have been painted black, so we suspect that the excess power could be reduced somewhat.

The sum of the internal radiation loads from the cryostat and JFETs is detected in noise spectra and contributes an NEP of $7 \times 10^{-17} \mathrm{~W} \mathrm{~Hz}^{-1 / 2}$. For the case of the cryostat at $4 \mathrm{~K}$, the more fundamental sources of detector noise have a minimum NEP of $8 \times 10^{-17} \mathrm{~W} \mathrm{~Hz}^{-1 / 2}$, resulting in a sensitivity limit of $1.1 \times 10^{-16} \mathrm{~W} \mathrm{~Hz}^{-1 / 2}$. For the purposes of SHARC II, we do not plan to address the $7 \times 10^{-17} \mathrm{~W} \mathrm{~Hz}^{-1 / 2} \mathrm{NEP}$ contamination since the background NEP in operation at $350 \mu \mathrm{m}$ is $\geq 6 \times 10^{-16} \mathrm{~W} \mathrm{~Hz}^{-1 / 2}$.

\subsection{Quantum efficiency}

We measured the quantum efficiency for a radiation load filling the field of view and for a source filling only a fraction of the field of view. At $350 \mu \mathrm{m}$, the observed background radiation load in the lab (emissivity of 1) is $230 \mathrm{pW}$, within $5 \%$ of the expected load calculated using the known filter profile (Figure 6), field of view of $0.042 \mathrm{sr}$, pixel area of $1 \mathrm{~mm}^{2}$, and assuming perfect detector absorption. When a small source - Eccosorb AN-72 foam dipped in liquid nitrogen - is used as the source, the derived detector quantum efficiency is only $\sim 75 \%$. The source of the discrepancy is unknown. In our subsequent calculations, we assume that the detector quantum efficiency is $100 \%$.

\subsection{Noise at low frequencies}

A noise spectrum with the instrument looking at a high-emissivity room temperature load is shown in Figure 7 . The spectrum is flat down to $<0.05 \mathrm{~Hz}$ and has the expected photon noise level, indicating good performance in 


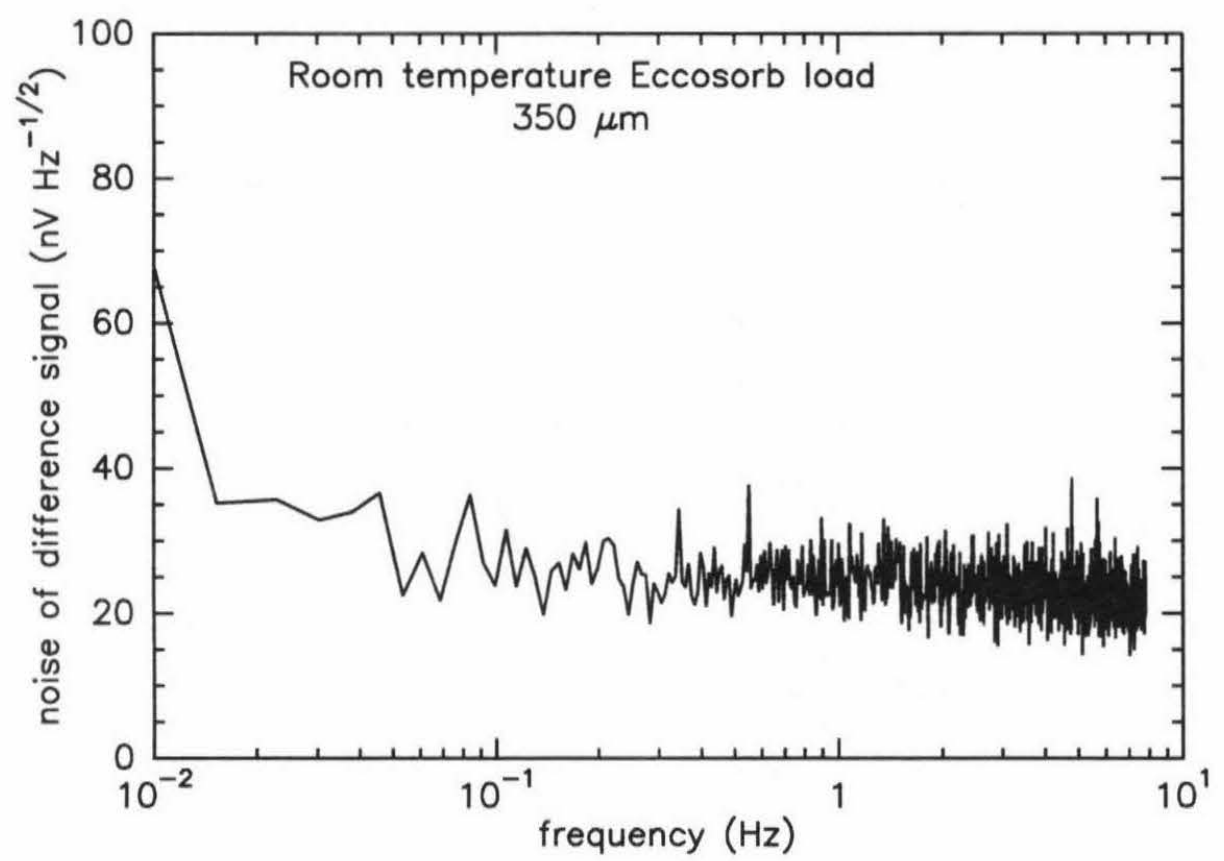

Figure 7. Noise spectrum for SHARC II observing room temperature Eccosorb foam. Even though shielding the foam from air currents significantly reduces correlated low-frequency noise over the array that we interpret as temperature drifts of the Eccosorb, there are still residual drifts with shielding. We show the power spectral density of the difference of two bolometer signals to subtract the residual correlated noise.

the total-power readout. On the telescope, the instrument shows no significant microphonic noise in the $\sim 30 \mathrm{~Hz}$ bandpass.

\section{OBSERVING MODES}

The baseline SHARC II observing modes are carried out with the secondary mirror of the CSO stationary. There are several advantages for this; among these is the reduction of image reconstruction artificats - specifically, the 'ghost' images at multiples of the chopper throw away from bright sources.

\subsection{Nodding modes}

If a camera has no excess low frequency noise on nodding time scales, then a small, deep field can be mapped by nodding the telescope back-and-forth without chopping. Since it involves a difference, this observing mode increases the fundamental noise by a factor of $\sqrt{2}$ (if the field is always on the detector array), so we assign it a factor of $\mathrm{C}^{\prime}=1.414$ in Equation 3 (§9.2). For comparison, a single detector with chopping and nodding results in $\mathrm{C}^{\prime}=2$.

\subsection{Scanning modes}

For sources comparable in size to or larger than the detector array, one can employ scanning observing modes. In principle, signals from astronomical sources can be separated from variations in the atmospheric emission due to their different spatial and temporal signatures. The scanning concept for SHARC II is similar to 'fastscanning' tested at the $30 \mathrm{~m}$ IRAM telescope. ${ }^{19}$ Differences are that the low $1 / \mathrm{f}$ noise of the AC-biased SHARC II readout and the absence of a high-pass filter allow slower scan rates. Drift scans (with the telescope stationary) with SHARC II at $350 \mu \mathrm{m}$ produce spatial signals at frequencies up to $4 \mathrm{~Hz}$, while controlled scan rates of up to $60^{\prime \prime} / \mathrm{sec}$ can be used without significant signal attenuation by the typical bolometer time constant $(\S 2.1)$ 


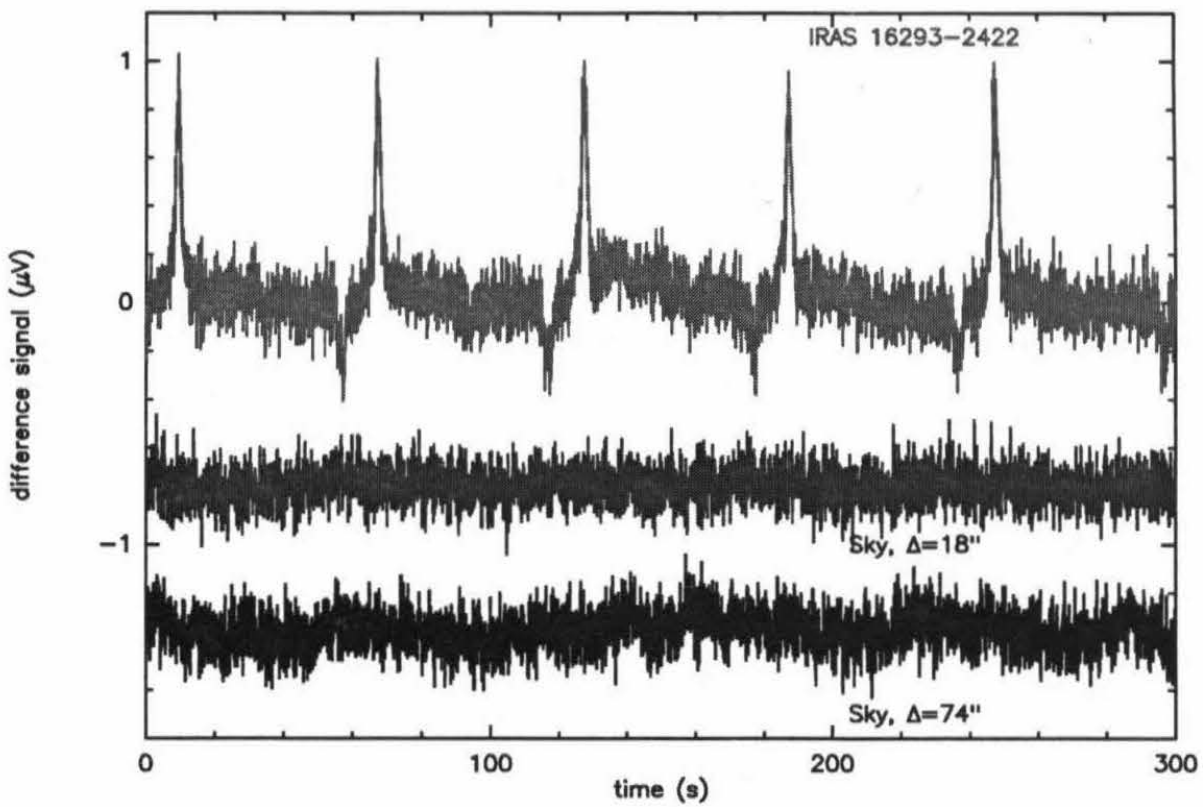

Figure 8. Signals from IRAS 16293-2422 (top trace) and nearby sky (bottom traces) derived from a difference of two bolometers as the telescope executed five cycles of a periodic, elliptical scan pattern. The difference is performed in software, and the signals are divided by the amplfier gain. The bolometers are $74^{\prime \prime}$ apart for the top and bottom traces and $18^{\prime \prime}$ apart for the middle trace. The negative deflection is not equal to the positive one (upper trace) since the scan direction is not along the line between the two bolometers.

and amplifier roll-off $(\S 4.2)$. Figure 8 illustrates characteristic signals obtained during a scanned observation without chopping.

\subsection{Calibration tools}

The voltage response $\mathrm{V}$ from a point source detected by a bolometer can be written as:

$$
\mathrm{V}=\mathrm{S} \eta \mathrm{A} \int \mathrm{d} \nu \mathrm{F}(\nu) \mathrm{e}^{-\tau(\nu)} \mathrm{Q}(\nu),
$$

where $\mathrm{S}$ is the bolometer responsivity (volts per watt), $\eta$ is the (geometrical) fraction of source power on the detector(s) compared to what could have been obtained with perfect imaging, $\mathrm{A}$ is the telescope area, $\mathrm{F}$ is the source flux density, $\mathrm{e}^{-\tau}$ is the atmospheric transmission, and $\mathrm{Q}$ is the instrument quantum efficiency. The terms which are variable during a night of observing are $\tau(\nu), \mathrm{S}$, and possibly $\eta$. In this section, we discuss procedures for quantifying $\tau(\nu)$ and S.

The traditional ways to measure the atmospheric opacity are to generate an extinction curve (multiple observations of a source at different airmasses), to perform a sky dip (observations of the sky at a series of airmasses), or to rely on a separate atmospheric monitor such as the $225 \mathrm{GHz} \mathrm{CSO}$ taumeter. All of these methods are available for SHARC II. In addition, we hope to use the total power capability of SHARC II to track changes in sky emission - and hence transmission - in real time.

To mitigate the problem of drift in bolometer responsivity S (e.g., due to changes in atmospheric loading), SHARC II has an internal calibration source in the vicinity of the detector array (Figure 5). The source is an 'inverse bolometer' with a $1 \mathrm{~mm}^{2}$ surface area and $<100 \mathrm{msec}$ time constant (Haller-Beeman Associates). Responsivity calibration integrations are separate from source integrations and relatively short. 
Absolute calibration of SHARC II is based on Mars, Uranus, and other sources which have been calibrated against them. ${ }^{20}$ We plan to reexamine (with $9^{\prime \prime}$ resolution) and extend the list of secondary calibrators at $350 \mu \mathrm{m}$ since many are extended sources which have been observed with coarser angular resolution.

\section{COMMISSIONING AT CALTECH SUBMILLIMETER OBSERVATORY}

\subsection{Software}

During the 2002 April and July engineering runs, SHARC II data were acquired with a JAVA program written inhouse which provides a real-time display of the bolometer samples and archives the samples, telescope position, instrument status, and observing conditions in FITS format. A future goal is to operate SHARC II via Goddard's Instrument Remote Control (IRC) environment. ${ }^{21}$ At the end of the July run, samples were successfully displayed and archived in IRC.

\subsection{Expected Sensitivity}

At $350 \mu \mathrm{m}$, SHARC II should have noise only $\sim 5 \%$ above background-limited operation calculated following, e.g., Mather. ${ }^{22}$ We find that the temporal correlation of photons approximately doubles the photon noise, compared to the Poisson noise term only, due to the high quantum efficiency and atmospheric opacity.

The NEP for a single detector (itself depending on bandpass and atmospheric conditions) can be converted to NEFD in the following way:

$$
\mathrm{NEFD}=\frac{\mathrm{C} \text { NEP }}{\eta A \int \mathrm{d} \nu \mathrm{e}^{-\tau(\nu)} \mathrm{Q}(\nu)}
$$

where $\mathrm{C}$ describes the observing technique, and $\mathrm{A}=79 \mathrm{~m}^{2}$. We assume the differencing observing mode without chopping ( $\S 8.1)$ and that four pixels are co-added: $\mathrm{C}=\sqrt{2} \times 2$. We decompose $\eta$ into three terms: $\eta_{\text {telescope }}=$ telescope aperture efficiency $=30 \%, \eta_{\text {diffraction }}=$ fraction of Airy pattern incident on 4 pixels $=$ $70 \%$, and $\eta_{\text {optics }}=$ Strehl ratio due to geometrical optical aberrations $=95 \%$. Then $\eta=20 \%$. For $\tau(\nu)$, we use atmospheric models from ATM. ${ }^{18}$ For Q, we use the measured profile from Figure 6 . The calculation results for the background-limited NEFD are shown in Figure 9. The NEFD is converted to a flux uncertainty obtained in time $\mathrm{t} \mathrm{via}^{23}$ :

$$
\sigma(\mathrm{F})=\frac{\mathrm{NEFD}}{\sqrt{2 \mathrm{t}}} .
$$

\subsection{Observed Sensitivity}

Using observations from the SHARC II engineering run in July 2002, we derive a measurement of the NEFD. IRAS 16293-2422 was observed with $\tau_{225 \mathrm{GHz}}($ zenith $)=0.068$ and at an airmass of 1.42 . The 'sky' signals (Figure 8) were Fourier transformed and converted to an NEFD spectrum (Figure 10) with the peak signal and known flux ${ }^{20}$ of IRAS $16293-2422$, using the optimum method of coadding four pixels. The NEFD in the stated weather conditions for modulation frequencies of $\sim 1 \mathrm{~Hz}$ is measured to be $4 \mathrm{Jy} \mathrm{Hz}^{-1 / 2}$, close to the calculated value for background-limited performance (Figure 9). The NEFD measurement extrapolates to $\lesssim 1 \mathrm{Jy} \mathrm{Hz}^{-1 / 2}$ in the best $25 \%$ of winter weather.

\subsection{Research plans}

We are just starting to map sources with SHARC II a month before this conference (Figure 11). Our plan is for SHARC II to become a facility available to all CSO users in February 2003, with observing proposals submitted in Fall 2002. We anticipate studies of molecular cloud cores and the environments of young and old stars, low-redshift galaxy mapping, $350 \mu \mathrm{m}$ follow-up of known high-z galaxies, and $350 \mu \mathrm{m}$ 'blank field' surveys. With an NEFD at least a factor of 2 better, and a field of view 7 times larger, SHARC II should be $30+$ times faster at mapping fields than its predecessor SHARC. ${ }^{1}$ 


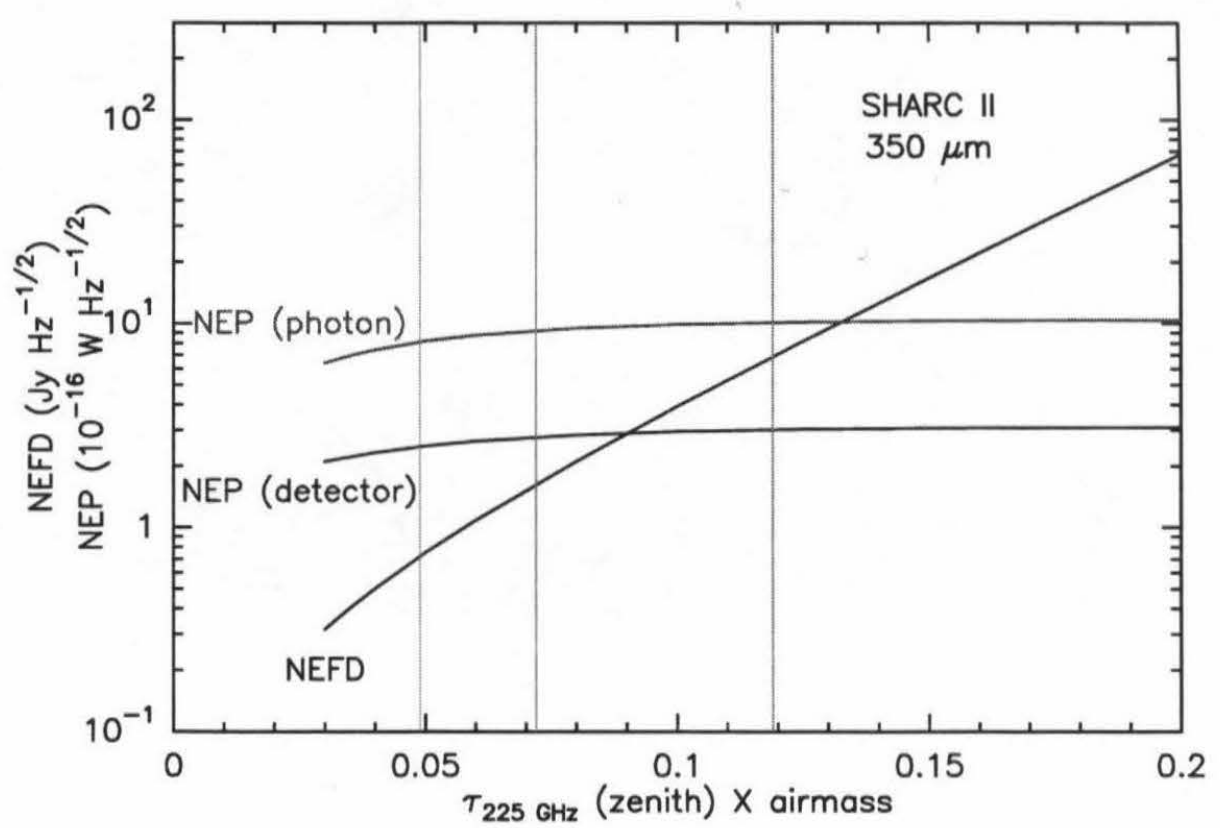

Figure 9. Photon-noise NEP, detector NEP, and background-limited NEFD as a function of atmospheric opacity. The photon NEP is fairly constant over the range of atmospheric conditions so that the slope of the NEFD is dominated by the atmospheric extinction $\tau(350 \mu \mathrm{m}) \approx 25 \tau_{225 \mathrm{GHz}}$. The detector NEP (which includes the $7 \times 10^{-17} \mathrm{~W} \mathrm{~Hz}^{-1 / 2}$ from internal radiation; §7.1) is approximately one third of the photon NEP, meaning that it inflates the system noise by only $\sim 5 \%$. The vertical lines show the quartiles of the Mauna Kea atmospheric opacity distribution for winter nights.

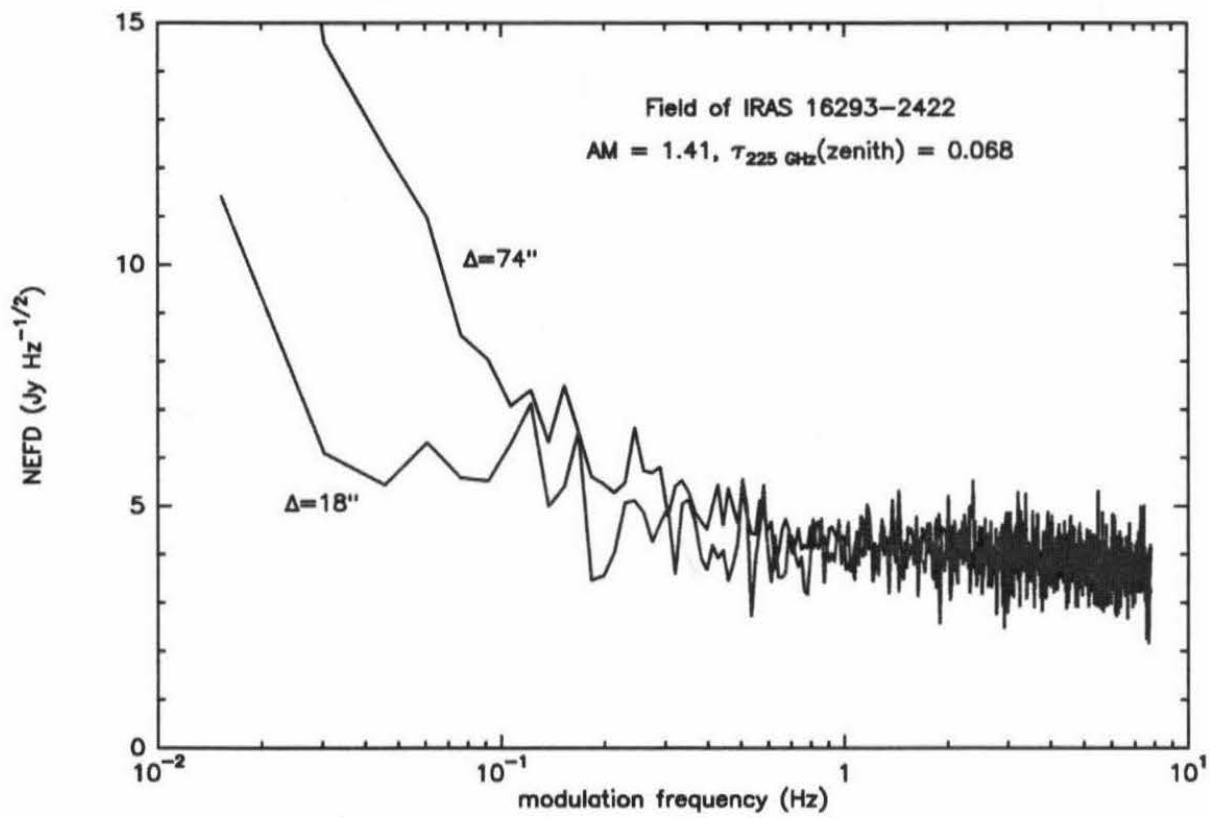

Figure 10. Observed NEFD for SHARC II at $350 \mu \mathrm{m}$ for a scanned observing mode without chopping. The $1 / \mathrm{f}$ noise is lower for pixels separated by $18^{\prime \prime}$ (bottom trace) than it is for pixels separated by $74^{\prime \prime}$ (top trace), which we attribute to variable gradients in sky emission. 


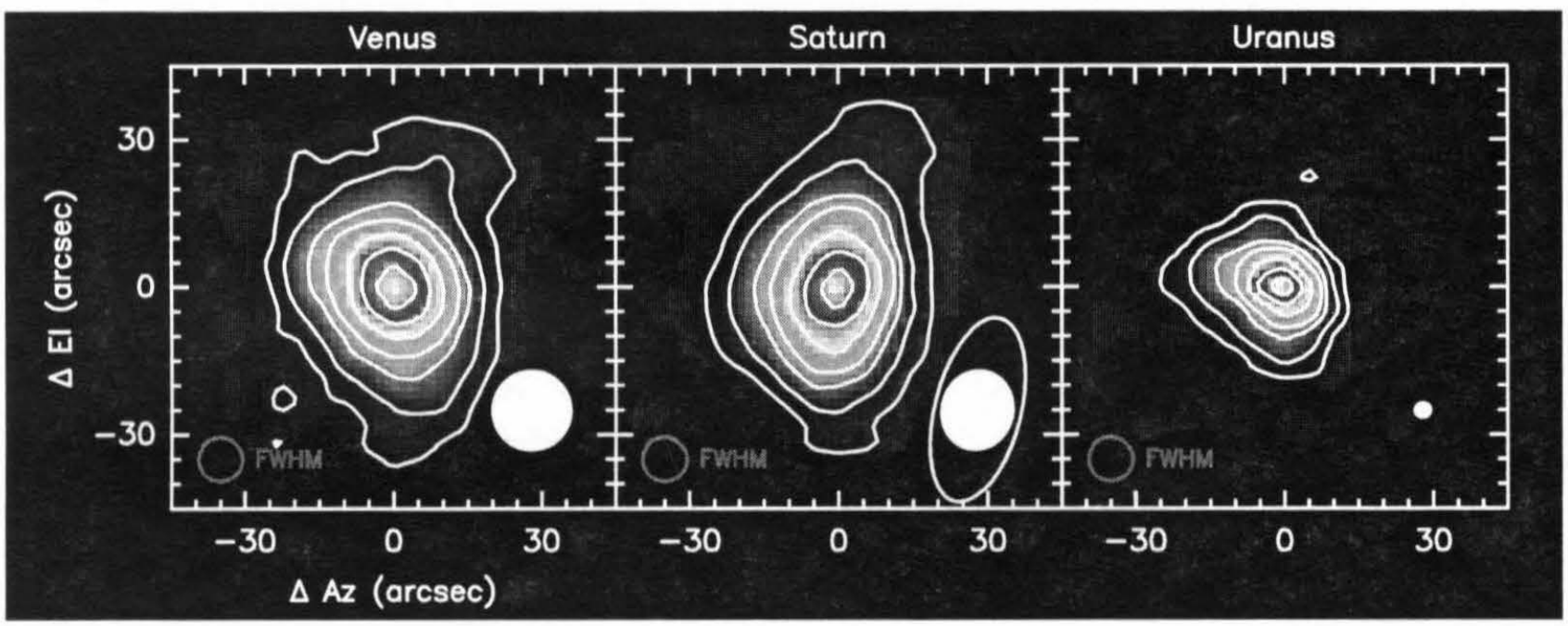

Figure 11. SHARC II $350 \mu \mathrm{m}$ images of planets from July 2002. The contour intervals are $90 \%, 70 \%, 50 \%, 30 \%, 20 \%$, $10 \%$, and $5 \%$. The disks at lower right show the physical extent of the planets at the time of observation. Venus was observed at 2.1 airmasses, Saturn at 1.7 airmasses, and Uranus at 1.5 airmasses.

\section{ACKNOWLEDGMENTS}

The CSO is funded by NSF grant AST 9980846. A large number of people at Goddard, Caltech, and elsewhere have contributed to the development of SHARC II, including: T. Ames, A. Bartels, D. Benford, W. Collins, B. Derro, J. Dotson, J. Foster, M. Gould, D. A. Harper, J. Higinbotham, Johns Hopkins Applied Physics Lab, K. Lewis, S. Lin, P. Nelson, R. Paniagua, F. Peters, V. Ponce, D. Sandford, C. Sappington, J. Staguhn, D. Warden, and J. Wirth.

\section{REFERENCES}

1. N. Wang, T. R. Hunter, D. J. Benford, E. Serabyn, D. C. Lis, T. G. Phillips, S. H. Moseley, K. Boyce, A. Szymkowiak, C. Allen, B. Mott, and J. Gygax, "Characterization of a submillimeter high-angularresolution camera with a monolithic silicon bolometer array for the Caltech Submillimeter Observatory," Appl. Opt. 35, pp. 6629-6640, 1996.

2. W. S. Holland, E. I. Robson, W. K. Gear, C. R. Cunningham, J. F. Lightfoot, T. Jenness, R. J. Ivison, J. A. Stevens, P. A. R. Ade, M. J. Griffin, W. D. Duncan, J. A. Murphy, and D. A. Naylor, "SCUBA: a common-user submillimetre camera operating on the James Clerk Maxwell Telescope," Mon. Not. R. Astron. Soc. 303, pp. 659-672, 1999.

3. E. Kreysa, H. Gemünd, J. Gromke, C. G. Haslam, L. Reichertz, E. E. Haller, J. W. Beeman, V. Hansen, A. Sievers, and R. Zylka, "Bolometer array development at the Max-Planck-Institut für Radioastronomie," in Advanced Technology MMW, Radio, and Terahertz Telescopes, T. G. Phillips, ed., Proc. SPIE 3357, pp. 319-325, 1998.

4. A. Omont, P. Cox, F. Bertoldi, R. G. McMahon, C. Carilli, and K. G. Isaak, "A 1.2 mm MAMBO/IRAM$30 \mathrm{~m}$ survey of dust emission from the highest redshift PSS quasars," Astron. Astrophys. 374, pp. 371-381, 2001.

5. F. Piacentini, P. A. R. Ade, R. S. Bhatia, J. J. Bock, A. Boscaleri, P. Cardoni, B. P. Crill, P. de Bernardis, H. Del Castillo, G. De Troia, P. Farese, M. Giacometti, E. F. Hivon, V. V. Hristov, A. Iacoangeli, A. E. Lange, S. Masi, P. D. Mauskopf, L. Miglio, C. B. Netterfield, P. Palangio, E. Pascale, A. Raccanelli, S. Rao, G. Romeo, J. Ruhl, and F. Scaramuzzi, "The BOOMERANG North America Instrument: A Balloon-borne Bolometric Radiometer Optimized for Measurements of Cosmic Background Radiation Anisotropies from 0.3 degree to 4 degrees," Astrophys. Jnl. Suppl. 138, pp. 315-336, 2002. 
6. A. T. Lee, P. Ade, A. Balbi, J. Bock, J. Borrill, A. Boscaleri, B. P. Crill, P. de Bernardis, H. del Castillo, P. Ferreira, K. Ganga, S. Hanany, V. Hristov, A. H. Jaffe, A. E. Lange, P. Mauskopf, C. B. Netterfield, S. Oh, E. Pascale, B. Rabii, P. L. Richards, J. Ruhl, G. F. Smoot, and C. D. Winant, "MAXIMA: An Experiment to Measure Temperature Anisotropy in the Cosmic Microwave Background," in $3 K$ Cosmology, AIP Conf. Proc. 476, pp. 224+, 1999.

7. D. A. Harper, C. A. Allen, M. J. Amato, T. Ames, A. Bartels, S. C. Casey, R. J. Derro, R. Evans, I. Gatley, S. J. Heimsath, A. Hermida, M. D. Jhabvala, J. H. Kastner, R. F. Loewenstein, S. H. Moseley, R. J. Pernic, T. Rennick, H. E. Rhody, D. Sandford, R. A. Shafer, P. J. Shirron, G. Voellmer, S. Wang, and J. Wirth, "HAWC: a far-infrared camera for SOFIA," in Airborne Telescope Systems, R. K. Melugin and H.-P. Roeser, eds., Proc. SPIE 4014, pp. 43-53, 2000.

8. M. J. Griffin, J. J. Bock, and W. K. Gear, "The Relative Performance of Filled and Feedhorn-Coupled Focal-plane Architectures," Appl. Opt. , accepted, 2002.

9. G. Voellmer, C. A. Allen, M. J. Amato, A. E. Bartels, D. J. Benford, R. J. Derro, C. D. Dowell, D. A. Harper, S. H. Moseley, Jr., P. J. Shirron, W. W. Smith, and J. G. Staguhn, "Design and fabrication of twodimensional semiconducting bolometer arrays for HAWC and SHARC-II," in Millimeter and Submillimeter Detectors for Astronomy, T. G. Phillips and J. Zmuidzinas, eds., Proc. SPIE 4855, 2002.

10. S.-I. Han, R. Almy, E. Apodaca, W. Bergmann, S. Deiker, A. Lesser, D. McCammon, K. Rawlins, R. L. Kelley, S. H. Moseley, F. S. Porter, C. K. Stahle, and A. E. Szymkowiak, "Intrinsic 1/f noise in doped silicon thermistors for cryogenic calorimeters," in EUV, X-Ray, and Gamma-Ray Instrumentation for Astronomy IX, O. H. Siegmund and M. H. Gummin, eds., Proc. SPIE 3445, pp. 640-644, 1998.

11. S. H. Moseley, Jr., C. D. Dowell, C. Allen, and T. G. Phillips, "Semiconducting Pop-Up Bolometers for Far-Infrared and Submillimeter Astronomy," in Imaging at Radio through Submillimeter Wavelengths, J. G. Mangum and S. J. E. Radford, eds., ASP Conf. Ser. 217, pp. 140-149, 2000.

12. M. M. Freund, R. F. Silverberg, V. Ponce, C. A. Allen, C. Sappington, S. H. Moseley, Jr., D. A. Harper, Jr., R. F. Loewenstein, and C. D. Dowell, "Testing and performance results for the $12 \times 32$ SHARC II and HAWC bolometer arrays," in Millimeter and Submillimeter Detectors for Astronomy, T. G. Phillips and J. Zmuidzinas, eds., Proc. SPIE 4855, 2002.

13. C. D. Dowell, J. E. Groseth, T. G. Phillips, C. A. Allen, S. R. Babu, M. D. Jhabvala, S. H. Moseley, Jr., and G. M. Voellmer, "The $12 \times 32$ pop-up bolometer array for the SHARC II camera," in Far-IR, SubMM \& MM Detector Technology Workshop, J. Wolf, J. Farhoomand, and C. R. McCreight, eds., NASA CP-211408, 2002.

14. M. Jhabvala, S. Babu, C. Monroy, M. M. Freund, and C. D. Dowell, "Development of low-noise high value chromium silicide resistors for cryogenic detector applications," Cryogenics, accepted, 2002.

15. G. Dall'Oglio, W. Fischer, L. Martinis, and L. Pizzo, "Improved ${ }^{3} \mathrm{He} /{ }^{4} \mathrm{He}$ refrigerator," Cryogenics 33, pp. 213-214, 1993.

16. E. Serabyn, "Wide-field Imaging Optics for Submm Arrays," in Multi-Feed Systems for Radio Telescopes, D. T. Emerson and J. M. Payne, eds., ASP Conf. Ser. 75, pp. 74-81, 1995.

17. T. R. Hunter, D. J. Benford, and E. Serabyn, "Optical Design of the Submillimeter High Angular Resolution Camera (SHARC)," Pub. Astron. Soc. Pac. 108, pp. 1042-1050, 1996.

18. J. R. Pardo, J. Cernicharo, and E. Serabyn, "Atmospheric Transmission at Microwaves (ATM): An Improved Model for mm/submm applications," IEEE Trans. on Antennas and Propagation 49, pp. 1683-1694, 2001.

19. L. A. Reichertz, B. Weferling, W. Esch, and E. Kreysa, "The fastscanning observing technique for millimeter and submillimeter astronomy," Astron. Astrophys. 379, pp. 735-739, 2001.

20. G. Sandell, "Secondary calibrators at submillimeter wavelengths.," Mon. Not. R. Astron. Soc. 271, pp. 7580, 1994 .

21. T. J. Ames and L. Case, "Distributed framework for dynamic telescope and instrument control," in Advanced Telescope and Instrumentation Control Software II, H. Lewis, ed., Proc. SPIE 4848, 2002.

22. J. C. Mather, "Bolometer noise: nonequilibrium theory," Appl. Opt. 21, pp. 1125-1129, 1982.

23. G. H. Rieke, Detection of Light: from the Ultraviolet to the Submillimeter, Cambridge University Press, Cambridge, 1994. 